\title{
Clinical Predictors for Saline Responsive Non-Edematous Hyponatremia among Hospitalized Patients
}

\author{
Naowanit Nata, Nardtaya Sriburin, Amnart Chaiprasert, Ouppatham Supasyndh and Bancha \\ Satirapoj* \\ Department of Medicine, Phramongkutklao Hospital and College of Medicine, Thailand
}

*Corresponding author: Bancha Satirapoj, 315, Division of Nephrology, Department of Medicine, Phramongkutklao Hospital and College of Medicine, Bangkok 10400, Thailand.

To Cite This Article: Naowanit Nata, Nardtaya Sriburin, Amnart Chaiprasert, Ouppatham Supasyndh, Bancha Satirapoj, Clinical Predictors for Saline Responsive Non-Edematous Hyponatremia among Hospitalized Patients. 2020 - 10(5). AJBSR.MS.ID.001549. DOI: 10.34297/ AJBSR.2020.10.001549.

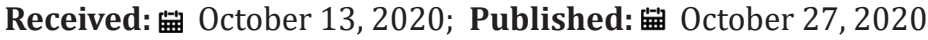

\begin{abstract}
Background: Hyponatremia is one of the most common electrolyte abnormalities in hospitals. Treatment of hyponatremia depends on symptoms and etiology related to volume status of the patients. Sometime evaluating volume status of patients is difficult and challenging for the physician to decide for therapeutic of choice.

Objective: This study was designed to determine the clinical and biochemical predictors for saline responsive hyponatremia.

Design and participants: We conducted a clinical study among hospitalized patients with no edematous hyponatremia. The clinical and laboratory data were collected by reviews of medical records and patients were classified in 2 groups: saline responder and saline no responder. Saline responder was defined as increased serum sodium $>5 \mathrm{mmol} / \mathrm{L}$ within 24 hours after saline treatment.

Results: Twenty-eight patients were saline responders (49.1\%), and 29 patients (50.9\%) were saline no responders. The mean serum sodium at baseline and 24 hours of therapy was $125.0 \pm 3.8$ and $132.0 \pm 3.8 \mathrm{mmol} / \mathrm{L}$, respectively, in the saline responder group and $125.5 \pm 4.4$ and $127.7 \pm 4.0$ $\mathrm{mmol} / \mathrm{L}$, respectively, in the saline no responder group. At baseline, the saline responder group had a higher incidence of dry mucosal membrane and lower estimated glomerular filtration rate, urine sodium and urine chloride than in the saline no responder group. From multivariate analysis, presence of dry mucosa showed a strong association with saline responder (Hazard ratio 8.17 with 95\% CI 1.05 to 63.39).
\end{abstract}

Conclusions: Dry mucosal membrane was a strong independent predictor for saline responders among hospitalized patients with hyponatremia. Further randomized control trial should confirm the findings.

Keywords: Saline responsive hyponatremia; Hyponatremia; Urine sodium; Hospitalized hyponatremia

\section{Introduction}

Hyponatremia is the most common of electrolyte disorders encountered in clinical practice [1] and is associated with increased morbidity, mortality and length of hospital stay among patients presenting a range of conditions [2-4]. Meta-analysis indicated that hyponatremia also relates to higher risk of re-admission and hospitalization costs [5]. Slow rate of correction in severe hyponatremia is associated with higher mortality [6]. Establishing etiology and appropriate treatment improves outcomes [7]. Clinical assessment of fluid status in the absence of diuretics, and a clinical assessment of volume status may aid further differential diagnosis.
Further, treating hyponatremia depends on symptoms and etiology related to volume status of patients.

An increase in plasma sodium is recommended to be limited to 10 to $12 \mathrm{mmol} / \mathrm{L}$ during the first 24 hours and to $8 \mathrm{mmol} / \mathrm{L} /$ day during the following days [8]. After hospitalization and diagnosis of hypotonic hyponatremia with no edematous patients, the physician must decide as soon as possible which treatment to choose such as saline infusion in hypovolemic hyponatremia. Volume resuscitation with isotonic saline in hyponatremia may result in an overshooting increase of plasma sodium because volume resuscitation quickly 
suppresses antidiuretic hormone (ADH) secretion [9]. Plasma sodium rises quickly; and therefore, careful monitoring of hypovolemic patients with hyponatremia receiving isotonic saline is mandatory. Clinical and biochemical parameters have been proposed to classify hyponatremic patients. In view of the limited efficacy of fluid treatment to correct hyponatremia in clinical practice, this study was designed to determine the clinical and biochemical parameters for predicting saline responsive after 24hour saline treatment in no edematous patients with hyponatremia.

\section{Material and Methods}

A prospective cohort study was carried out among patients with hyponatremia hospitalized at Phramongkutklao Hospital, Bangkok, Thailand between May 1, 2011 and March 31, 2013. The study was approved by the Ethics Committee of the Institute Review Board at the Royal Thai Army Medical Department and all subjects gave written informed consent.

\section{Subjects}

All diagnosed asymptomatic no edematous hyponatremia subjects with plasma osmolarity $<275 \mathrm{mOsm} / \mathrm{kg}$, plasma glucose $<200 \mathrm{mg} / \mathrm{dL}$, serum creatinine $<2 \mathrm{mg} / \mathrm{dL}$, plasma sodium concentration between 115 and $134 \mathrm{mmol} / \mathrm{L}$ and age more than 18 years were included in the study. All subjects received saline treatment at initial resuscitation. Patients were ineligible if they had clinically evident hypervolemic and euvolemic hyponatremia such as congestive heart failure, cirrhosis, advanced chronic kidney disease, adrenal, thyroid, or pituitary insufficiency, nephrotic syndrome or severe symptomatic hyponatremia. Other exclusion criteria were recently received oral or intravenous saline before admission Study assessments. The patients were divided in two groups (saline responsive and saline nonresponsive groups). Saline responsive hyponatremia was defined as increased equally or more than $5 \mathrm{mmoL}$ of sodium per liter after receiving $2 \mathrm{~L}$ of saline treatment within 24 hours.

A detailed medical history was collected by reviewing medical records of the hospitals of all patients. Clinical data collected at the onset of hyponatremia included age, sex, body weight, height, underlying diseases, medications, clinical status, and symptoms. Physical examination at admission included vital signs, postural hypotension, dry lips and tongue, skin turgor, jugular venous pressure, lung crepitation, ascites and edema. Plasma biochemical data collected at the onset of hyponatremia and after saline treatment included blood urea nitrogen (BUN), serum osmolarity, creatinine, electrolytes, uric acid, phosphate, magnesium, free thyroxine and thyroid stimulating hormone. Urine biochemical data were also measured for urine osmolarity, electrolytes, creatinine, uric acid, urea, phosphate and magnesium at baseline and 24 hours of treatment.

\section{Statistical analysis}

Descriptive data were examined for all variables. For continuous variables, results are presented as mean \pm SD. Statistical differences in variables were compared using one-way analysis of variance, unpaired Student's t-test for normally distributed variables and Kruskal-Wallis Test for nonnormally distributed variables. Categorical variables were recorded as frequency counts, and intergroup comparisons were analyzed by chi-squared test. Associations between saline responsive, clinical and biochemical data were analyzed using multivariate logistic regression analysis [Hazard ratio (HR) with 95\% confidence intervals (CI)] and multivariate analyses were conducted after including variables such as age, systolic blood pressure, dry mucosal membrane, serum chloride, serum bicarbonate, BUN, estimated glomerular filtration rate (eGFR) by CKD-EPI equation, urine sodium and urine chloride. Statistical significance was accepted for $\mathrm{P}<0.05$. Data analysis was performed using SPSS for Windows, Version 12.0 (SPSS, Chicago, IL, USA).

\section{Results}

Four hundred and eighty patients with hyponatremia at Phramongkutklao Hospital between May 1, 2011 and March 31, 2013 were screened. After excluding ineligible records, a total of 57 were enrolled for analysis and classified in saline responsive and saline nonresponsive groups. In all, 28 subjects were in the saline responsive group and 29 subjects in the saline nonresponsive group. All patients were elderly Thais. At hospital discharge, final diagnosis of no edematous hyponatremia was hypovolemic hyponatremia from extrarenal and renal wasting $(\mathrm{N}=49)$ and syndrome of inappropriate secretion of antidiuretic hormone $(\mathrm{N}=8)$. Baseline characteristics of the subjects were similar in both groups (Table 1).

Table 1: Characteristics between saline responsive and saline nonresponsive groups.

\begin{tabular}{|c|c|c|c|}
\hline Characteristics & Saline responsive group $(\mathrm{N}=28)$ & Saline non-responsive group $(\mathrm{N}=29)$ & P-value \\
\hline Age (years) & $70.6 \pm 14.7$ & $65.5 \pm 16.4$ & 0.222 \\
\hline Gender (male) (n, \%) & $14(50.0 \%)$ & $18(62.1 \%)$ & 0.429 \\
\hline Hypertension (n, \%) & $11(39.3 \%)$ & $11(37.9 \%)$ & 1 \\
\hline Type 2 diabetes (n, \%) & $4(14.3 \%)$ & $4(13.8 \%)$ & 1 \\
\hline Malignancy (n, \%) & $8(28.6 \%)$ & $5(17.2 \%)$ & 0.358 \\
\hline
\end{tabular}




\begin{tabular}{|c|c|c|c|}
\hline Infection (n, \%) & $10(35.7 \%)$ & $12(41.4 \%)$ & 0.787 \\
\hline History of diuretics (n, \%) & $4(14.3 \%)$ & $2(6.9 \%)$ & 0.423 \\
\hline History of volume loss (n, \%) & $5(17.9 \%)$ & $2(6.9 \%)$ & 0.253 \\
\hline
\end{tabular}

Note: Categorical variables expressed as number (\%) and $\chi 2$ test for $P$ value. Continuous variables expressed as mean \pm SD and unpaired Student's t-test for $\mathrm{P}$ value between groups.

Clinical parameters of both groups are shown in Table 2. The values at baseline including systolic blood pressure, diastolic blood pressure, pulse rate, body temperature, respiratory rate and sunken eye ball did not significantly differ between the two groups except incidence of dry mucosal membrane was higher in the saline responsive group than that of the saline nonresponsive group ( 46.4 vs. $13.8 \%, \mathrm{P}=0.008$ ). The mean serum sodium at baseline and 24 hours of therapy was $125.0 \pm 3.8$ and $132.0 \pm 3.8$ $\mathrm{mmol} / \mathrm{L}$, respectively, in the saline responder group and $125.5 \pm 4.4$ and $127.7 \pm 4.0 \mathrm{mmol} / \mathrm{L}$, respectively, in the saline nonresponder group. The mean concentration at baseline of saline responsive and saline nonresponsive groups was: plasma osmolarity $265.8 \pm 12.0$ and $265.2+12.3 \mathrm{mOsm} / \mathrm{kg}$, urine osmolality $369.0 \pm 154.9$ and $435.7 \pm 169.0 \mathrm{mOsm} / \mathrm{kg}$, urine sodium $38.8 \pm 26.2$ and $63.2 \pm 46.8$ $\mathrm{mmol} / \mathrm{L}$ and fractional excretion of filtered sodium (FENa) $0.7 \pm 0.6$ and $0.7 \pm 0.5 \%$, respectively. The saline responsive group had significantly lower estimated GFR $(70.7 \pm 25.3$ vs. $87.4 \pm 27.0 \mathrm{~mL} /$ $\min / 1.73 \mathrm{~m} 2, \mathrm{P}=0.024)$, urine sodium $(38.8 \pm 26.2$ vs. $63.2 \pm 46.8$ $\mathrm{mmol} / \mathrm{L}, \mathrm{P}=0.020)$ and urine chloride $(32.8 \pm 29.5$ vs. $61.9 \pm 45.3$, $\mathrm{P}=0.016$ ) at baseline than the saline nonresponsive group (Table 3).

Table 2: Clinical parameters between saline responsive and saline nonresponsive groups at baseline.

\begin{tabular}{|c|c|c|c|}
\hline Parameters & Saline responsive group $(\mathrm{N}=28)$ & Saline non-responsive group $(\mathrm{N}=29)$ & P-value \\
\hline Systolic blood pressure (mmHg) & $130.3 \pm 26.9$ & $120.2 \pm 17.0$ & 0.096 \\
\hline Diastolic blood pressure (mmHg) & $71.3 \pm 12.9$ & $73.7 \pm 11.9$ & 0.467 \\
\hline Pulse rate (beat per minute) & $92.0 \pm 20.0$ & $96.0 \pm 17.0$ & 0.386 \\
\hline Body temperature $\left({ }^{\circ} \mathrm{C}\right)$ & $36.2 \pm 0.4$ & $36.9 \pm 0.9$ & 0.551 \\
\hline Respiratory rate (per minute) & $21.0 \pm 3.0$ & $21.0 \pm 5.0$ & 0.69 \\
\hline Dry mucosal membrane & $13(46.4 \%)$ & $4(13.8 \%)$ & 0.008 \\
\hline Sunken eyeball & $2(7.1 \%)$ & $1(3.5 \%)$ & 0.61 \\
\hline Poor skin turgor & $3(10.7 \%)$ & $0(0 \%)$ & 0.111 \\
\hline
\end{tabular}

Note: Categorical variables expressed as number (\%) and $\mathrm{X} 2$ test for $\mathrm{P}$ value. Continuous variables expressed as mean \pm SD and unpaired Student's t-test for $P$ value between groups.

Table 3: Laboratory parameters between saline responsive and saline nonresponsive groups at baseline and 24 hours.

\begin{tabular}{|c|c|c|c|}
\hline Parameters & Saline responsive group $(\mathrm{N}=28)$ & Saline non-responsive group $(\mathrm{N}=29)$ & P-value \\
\hline \multicolumn{4}{|c|}{ Baseline investigation } \\
\hline Plasma osmolarity (mOsm/kg) & $265.8 \pm 12.0$ & $265.2 \pm 12.3$ & 0.866 \\
\hline Plasma glucose (mg/dL) & $117.7 \pm 22.5$ & $125.7 \pm 32.9$ & 0.347 \\
\hline Blood urea nitrogen (mg/dL) & $20.4 \pm 11.0$ & $18.6 \pm 11.9$ & 0.589 \\
\hline Estimated GFR (mL/min/1.73 m²) & $70.7 \pm 25.3$ & $87.4+27.0$ & 0.024 \\
\hline Serum creatinine $(\mathrm{mg} / \mathrm{dL})$ & $1.0 \pm 0.4$ & $0.8 \pm 0.4$ & 0.106 \\
\hline Plasma sodium (mmol/L) & $125.0 \pm 3.8$ & $125.5 \pm 4.4$ & 0.657 \\
\hline Plasma potassium (mmol/L) & $3.7 \pm 0.8$ & $4.0 \pm 0.5$ & 0.142 \\
\hline Plasma chloride (mmol/L) & $90.4 \pm 5.5$ & $90.6 \pm 6.4$ & 0.922 \\
\hline Plasma HCO3 (mmol/L) & $22.6 \pm 3.2$ & $23.6 \pm 3.8$ & 0.311 \\
\hline Urine osmolarity (mOsm/kg) & $369.0 \pm 154.9$ & $435.7 \pm 169.0$ & 0.232 \\
\hline Urine creatinine (mg/dL) & $78.2 \pm 70.1$ & $66.9 \pm 38.3$ & 0.496 \\
\hline Urine sodium (mmol/L) & $38.8 \pm 26.2$ & $63.2 \pm 46.8$ & 0.02 \\
\hline Urine potassium (mmol/L) & $32.3 \pm 19.8$ & $31.0 \pm 15.5$ & 0.811 \\
\hline Urine chloride (mmol/L) & $32.8 \pm 29.5$ & $61.9 \pm 45.3$ & 0.016 \\
\hline Urine specific gravity & $1.014 \pm 0.006$ & $1.016 \pm 0.005$ & 0.116 \\
\hline
\end{tabular}




\begin{tabular}{|c|c|c|c|}
\hline Fractional excretion sodium (FE Na) (\%) & $0.7 \pm 0.6$ & $0.7 \pm 0.5$ \\
\hline \multicolumn{2}{|c|}{ 24-hours after normal saline treatment } \\
\hline Blood urea nitrogen (mg/dL) & $19.7+9.6$ & $17.3 \pm 10.8$ & 0.918 \\
\hline Serum creatinine (mg/dL) & $0.9 \pm 0.3$ & $0.9 \pm 0.6$ & 0.986 \\
\hline Plasma sodium (mmol/L) & $132.0 \pm 3.8$ & $327.7 \pm 4.0$ & $<0.001$ \\
\hline Plasma potassium (mmol/L) & $3.9 \pm 0.6$ & $93.9 \pm 5.9$ & 0.452 \\
\hline Plasma chloride (mmol/L) & $98.3 \pm 5.3$ & $22.9 \pm 3.4$ & 0.005 \\
\hline Plasma HCO3 (mmol/L) & $22.4 \pm 3.8$ & 0.536 \\
\hline
\end{tabular}

Note: Continuous variables expressed as mean \pm SD and unpaired Student's t-test for $P$ value between groups.

Using univariable analysis, dry mucosal membrane [HR 5.75 (95\% 1.55 to 21.33], estimated GFR [HR 0.98 (95\% CI 0.96-0.99)], urine sodium [HR 0.98 (95\% CI 0.97-0.99)] and urine chloride [HR 0.98 (95\% CI 0.96-0.99)] were significant associated with saline response treatment. In multivariable-adjusted analyses, only dry

Table 4: Predictive factors for saline responsive hyponatremia.

\begin{tabular}{|c|c|c|c|c|}
\hline Variables & Univariate analysis & \multicolumn{2}{|c|}{ Multivariate analysis } \\
\hline & Hazard ratio (95\% CI) & P-value & Hazard ratio (95\% CI) & P-value \\
\hline Age (years) & $1.02(0.99-1.06)$ & 0.222 & $1.00(0.93-1.08)$ & 0.992 \\
\hline Dry mucosal membrane & $5.75(1.55-21.33)$ & 0.009 & $8.17(1.05-63.39)$ & 0.045 \\
\hline Systolic blood pressure (mmHg) & $1.02(0.99-1.05)$ & 0.099 & $1.01(0.97-1.06)$ & 0.557 \\
\hline Blood urea nitrogen (mg/dL) & $1.01(0.97-1.06)$ & 0.582 & $1.09(0.92-1.30)$ & 0.314 \\
\hline Estimated GFR (mL/min/1.73 m $\left.{ }^{2}\right)$ & $0.98(0.96-0.99)$ & 0.029 & $1.02(0.96-1.09)$ & 0.494 \\
\hline Plasma chloride (mmol/L) & $0.99(0.91-1.09)$ & 0.921 & $0.90(0.67-1.21)$ & 0.493 \\
\hline Plasma bicarbonate (mmol/L) & $0.92(0.79-1.08)$ & 0.306 & $1.04(0.68-1.58)$ & 0.871 \\
\hline Urine chloride (mmol/L) & $0.98(0.96-0.99)$ & 0.036 & $1.00(0.96-1.04)$ & 0.861 \\
\hline Urine sodium (mmol/L) & $0.98(0.97-0.99)$ & 0.032 & $0.99(0.94-1.04)$ & 0.729 \\
\hline Pulse rate & $0.99(0.96-1.02)$ & 0.38 & & \\
\hline FE Na & $0.96(0.42-2.17)$ & 0.916 & & \\
\hline
\end{tabular}

Note: Associations between saline responsive, clinical and biochemical data were analyzed using multivariate logistic regression analysis [Hazard ratio $(\mathrm{HR})$ with $95 \%$ confidence intervals $(\mathrm{Cl})$ ] and multivariate analyses were conducted after including variables such as age, systolic blood pressure, dry mucosal membrane, serum chloride, serum bicarbonate, BUN, estimated glomerular filtration rate (eGFR) by CKD-EPI equation, urine sodium and urine chloride. Statistical significance was accepted for $\mathrm{P}<0.05$.

\section{Discussion}

Our data demonstrated that a significant proportion of a well-defined, prospective cohort of patients with asymptomatic hyponatremia had clinical hypovolemia from dry mucosal membrane and decreased renal perfusion from low urine sodium and chloride that could predict response of initial fluid therapy. This might explain why the observations on clinical efficacy of fluid treatment in the clinical study of hypovolemic hyponatremia resulted in an overshooting increase of plasma sodium, from suppressing ADH effects. Assessing effective arterial blood volume has an essential role in evaluating the cause and determining the adequate treatment of patients with hyponatremia. Patients with clinical signs of volume depletion such as orthostatic decreases in blood pressure, increases in pulse rate, dry mucus membrane mucosal membrane [HR 8.17 (95\% 1.05-63.39)] was independently associated with saline response treatment $(\mathrm{P}=0.045)$ controlling for age, systolic blood pressure, BUN, estimated GFR, plasma chloride, bicarbonate, urine sodium, urine chloride, pulse rate and FE Na (Table 4). 
In hypovolemic states with proper renal function, urinary sodium concentration values were low, typically less than 20 mmol/L [13] and low urinary sodium concentration clearly separated hypovolemic from normovolemic hyponatremic patients [14]. Therefore, urine sodium, and occasionally, urine chloride concentration is used as laboratory parameters to predict saline response $[14,15]$. Urine sodium at 20 to $30 \mathrm{mmol} / \mathrm{L}$ was used as a cutoff value to determine pattern response to isotonic saline infusion [16]. However, another study demonstrated that urine sodium levels up to $50 \mathrm{mmol} / \mathrm{L}$ showed high accuracy in separating SIADH from hypovolemic hyponatremia and had clinically meaningful responses to saline therapy [17]. This was consistent with our findings that only low urinary sodium at $38.8 \mathrm{mmol} / \mathrm{L}$ and chloride at $32.8 \mathrm{mmol} / \mathrm{L}$ were predictors for saline response. The difference due to the study population especially elderly patients in our study may have slowed adaptation to hypovolemia with reduced urinary sodium [18,19] and patients with renal tubular damage or renal salt wasting were observed, leading to volume contraction and increased urine sodium.

FENa is useful in evaluating patients with oliguria to distinguish between prerenal azotemia and acute kidney injury. FENa below $1 \%$ is an indicator of effective volume depletion [20]. One study demonstrated that low FENa $(<0.5 \%)$ combined with low fractional excretion of urea $(<55 \%)$ constituted a test to diagnose saline responsiveness [12]. However, some authors do not use FENa $<1 \%$ to define effective arterial blood volume depletion among patients with normal kidney function who have a much higher GFR and a much greater filtered sodium load [21]. This was consistent with our findings that level of FENa did not constitute a predictor for saline responsiveness.

The usefulness of biochemical parameters was limited by the overlap among different etiologies of hyponatremia. Our study found that other biochemical results at the time of diagnosis were similar between response and nonresponse patients. Other biochemical indicators such as plasma urea and uric acid levels were influenced by extracellular fluid volume; however, several factors also affected them [22]. None of the best biochemical profiles proved to have a sufficiently high predictive value for saline response among hospitalized patients with hyponatremia. Our study also indicated that only dry mucosal membrane was independently associated with saline response treatment in the multivariable-adjusted analyses.

Our study encountered several limitations. As the ethics approval was for an observational study only, we had no influence over fluid therapy and other treatments; the therapies selected were so varied, that we could not test whether the subsequent response to normal saline treatment validated the advice in the current practical guidelines. This might be seen as a limitation of our results, so we required a randomized controlled prospective study to confirm the findings. At admission, all subjects were thought to have hypovolemic hyponatremia. However, based on the clinical course and further investigation, the final diagnosis of hypovolemic hyponatremia at the time of discharge was reduced to $70 \%$. The saline response pattern or the magnitude of increase in plasma sodium level depends on the final diagnosis of hyponatremia.

\section{Conclusion}

Our prospective cohort study indicated that asymptomatic hospitalized hyponatremia patients with clinical hypovolemia from dry mucosal membrane could predict response of initial fluid therapy. Further randomized control trial should confirm the findings.

\section{Acknowledgment}

The authors thank staffs in the Division of Nephrology, Department of Medicine, Phramongkutklao Hospital and College of Medicine, for their research contributions.

\section{Author Contributions}

Naowanit Nata conceptualized the study, conducted the data extraction, analysed the data and wrote the first draft of the manuscript. Nardtaya Sriburin and Bancha Satirapoj contributed to the conceptualisation of the study, critically edited and proofread the document. Amnart Chaiprasert and Ouppatham Supasyndh proofread the document.

\section{Ethics Approval}

This study followed the guidelines of the Declaration of Helsinki and was approved by the Ethics Committee of the Institute Review Board at the Royal Thai Army Medical Department ((R098h/57).

\section{References}

1. Upadhyay A, Jaber BL, Madias NE (2006) Incidence and prevalence of hyponatremia. Am J Med 119(7 Suppl 1): S30-35.

2. Wald R, Jaber BL, Price LL, Upadhyay A, Madias NE (2010) Impact of hospital-associated hyponatremia on selected outcomes. Arch Intern Med 170(3): 294-302.

3. Funk GC, Lindner G, Druml W, Metnitz B, Schwarz C, et al. (2010) Incidence and prognosis of dysnatremias present on ICU admission. Intensive Care Med 36(2): 304-311.

4. Gill G, Huda B, Boyd A, Skagen K, Wile D, et al. (2006) Characteristics and mortality of severe hyponatraemia--a hospital-based study. Clin Endocrinol (Oxf) 65(2): 246-249.

5. Corona G, Giuliani C, Parenti G, Colombo GL, Sforza A, et al. (2016) The Economic Burden of Hyponatremia: Systematic Review and MetaAnalysis. Am J Med 129(8): 823-835.e824.

6. Nzerue CM, Baffoe-Bonnie H, You W, Falana B, Dai S (2003) Predictors of outcome in hospitalized patients with severe hyponatremia. J Natl Med Assoc 95(5): 335-343.

7. Sahay M, Sahay R (2014) Hyponatremia: A practical approach. Indian J Endocrinol Metab 18(6): 760-771. 
8. Weismann D, Schneider A, Hoybye C (2016) Clinical aspects of symptomatic hyponatremia. Endocr Connect 5(5): R35-R43.

9. Henry DA (2015) In the Clinic: Hyponatremia. Ann Intern Med 163(3): ITC1-19.

10. Buffington MA, Abreo K (2016) Hyponatremia: A Review. J Intensive Care Med 31(4): 223-236.

11. Verbalis JG, Goldsmith SR, Greenberg A, Korzelius C, Schrier RW, et al (2013) Diagnosis, evaluation, and treatment of hyponatremia: expert panel recommendations. Am J Med 2013, 126(10 Suppl 1): S1-42.

12. Musch W, Thimpont J, Vandervelde D, Verhaeverbeke I, Berghmans T, et al. (1995) Combined fractional excretion of sodium and urea better predicts response to saline in hyponatremia than do usual clinical and biochemical parameters. Am J Med 99(4): 348-355.

13. Hoorn EJ, Zietse R (2017) Diagnosis and Treatment of Hyponatremia: Compilation of the Guidelines. J Am Soc Nephrol 28(5): 1340-1349.

14. Chung HM, Kluge R, Schrier RW, Anderson RJ (1987) Clinical assessment of extracellular fluid volume in hyponatremia. Am J Med 83(5): 905-908.

15. Decaux G, Musch W (2008) Clinical laboratory evaluation of the syndrome of inappropriate secretion of antidiuretic hormone. Clin J Am Soc Nephrol 3(4): 1175-1184.
16. Milionis HJ, Liamis GL, Elisaf MS (2002) The hyponatremic patient: a systematic approach to laboratory diagnosis. CMAJ 166(8): 1056-1062.

17. Hato T, Ng R (2010) Diagnostic value of urine sodium concentration in hyponatremia due to syndrome of inappropriate antidiuretic hormone secretion versus hypovolemia. Hawaii Med J 69(11):264-267.

18. Baylis C, Corman B (1998) The aging kidney: insights from experimental studies. J Am Soc Nephrol 9(4): 699-709.

19. Musch W, Decaux G (2001) Utility and limitations of biochemical parameters in the evaluation of hyponatremia in the elderly. Int Urol Nephrol 32(3): 475-493.

20. Perazella MA, Coca SG (2012) Traditional urinary biomarkers in the assessment of hospital-acquired AKI. Clin J Am Soc Nephrol 7(1): 167174.

21. Steiner RW (1984) Interpreting the fractional excretion of sodium. Am J Med 77(4): 699-702.

22. Decaux G, Schlesser M, Coffernils M, Prospert F, Namias B, et al. (1994) Uric acid, anion gap and urea concentration in the diagnostic approach to hyponatremia. Clin Nephrol 42(2):102-108. 